\title{
Prophylactic HIPEC in high-risk colorectal cancer: do we really need a randomized clinical trial to prove a "logical" hypothesis?
}

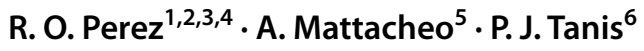

Published online: 18 April 2021

C) Springer Nature Switzerland AG 2021

Cytoreductive surgery with or without hyperthermic intraperitoneal chemotherapy has become a valid alternative for the treatment of peritoneal carcinomatosis in selected patients with colorectal cancer. While refinements in patient selection and in the understanding of the role of intraperitoneal chemotherapy are still being investigated in the setting of randomized controlled trials, the procedure has clearly provided hope to patients once considered to be at an endstage disease and with little survival perspective [1].

The optimism created by multiple series reporting surprisingly favorable outcomes with this approach for the metastatic setting led surgeons to consider the use of prophylactic hyperthermic intraperitoneal chemotherapy among patients with high risk for peritoneal dissemination colon cancer [2]. In theory, patients with colorectal cancers invading through the parietal peritoneum and, therefore, potentially seeding the peritoneal cavity could benefit from intraperitoneal chemotherapy to treat (or "prevent") microscopic and clinically undetectable peritoneal implants.

A prospective randomized clinical trial addressed this particular issue by comparing adjuvant systemic and intraperitoneal chemotherapy to systemic chemotherapy alone failed to show any significant differences in peritoneal

R. O. Perez

rodrigo.operez@gmail.com

1 Angelita and Joaquim Gama Institute, Praça Amadeu Amaral, 47, Conj.111, São Paulo, SP ZIP 01327-904, Brazil

2 Ludwig Institute for Cancer Research, São Paulo Branch, São Paulo, Brazil

3 Departamento de Cirurgia Oncológica, Hospital Beneficencia Portuguesa, São Paulo, Brazil

4 Hospital Alemão Oswaldo Cruz, São Paulo, Brazil

5 Hospital J. M. Ramos Mejía, Ciudad Autónoma de Buenos Aires, Argentina

6 Department of Surgery, Amsterdam University Medical Centers, University of Amsterdam, Cancer Centre Amsterdam, Amsterdam, The Netherlands metastases-free survival at 18 months from the procedure between groups [3]. In the present issue of Techniques in Coloproctology, Li et al. challenge the findings of this randomized clinical trial in a large retrospective (and rather similar) study. In their investigation, Li et al. report on the outcomes of a large series of patients managed by systemic chemotherapy alone compared to systemic chemotherapy and prophylactic HIPEC, showing meaningful and statistically significant differences in survival outcomes favoring prophylactic HIPEC. While the results here may seem intuitively logical at a first glance, readers should take a step back before they consider incorporating this approach into clinical practice.

One important potential source of selection bias may have definitively contributed for the differences observed between groups in this study. Allocation of patients to each treatment arm took into consideration an "economical" factor. Besides the significant ethical considerations in studies where economical factors influence allocation of patients to different treatment arms, this potential selection bias may have significantly affected results [4]. Ultimately, a significant proportion of patients originally allocated to one arm (HIPEC + systemic therapy) failed to receive treatment due to impossibility to deal with HIPEC-associated "costs". Even though this may look like a modest source of potential selection bias, it actually hides numerous relevant inherent differences between patients in each group. Patients "capable" of dealing with extra costs associated with the experimental prophylactic HIPEC arm are more likely to have been "better" candidates (clinically, nutritionally, etc.) than patients in whom such financial burden was significant, and therefore declined such treatment alternative. In addition, socioeconomic status per se, seems to be a driving force in survival after colorectal cancer [5]. Therefore, patients assigned to the experimental arm were clearly "different" from those where the only possibility was standard systemic chemotherapy. 
Intraperitoneal hyperthermic chemotherapy may seem perfectly logical for the prevention of peritoneal recurrence in high-risk colorectal cancer. Currently, additional ongoing trials are attempting to further elucidate the potential role of HIPEC in preventing peritoneal carcinomatosis among these patients. Until then, readers should be aware that less than properly designed studies, and biased allocation of patients, may actually misleadingly support and confirm the rather "logical" hypothesis. However, sometimes science proves us wrong and even the most plausible hypothesis is denied when proper control groups and adequate allocation methods are used. This is why the outcomes of well-designed ongoing clinical trials are so important before we even consider changing clinical practice $[1,6]$.

Until new available data are provided from well-designed clinical trials, prophylactic HIPEC should not be routinely offered to high-risk colorectal cancer, even among patients willing to accept the financial burden associated with it.

\section{Compliance with ethical Standards}

Conflict of interest The authors declare that they have no conflict of interest.

Ethical approval This article does not contain animal or human subjects involved in any part of the study and therefore does not require formal ethical approval.
Informed consent For this kind of study, formal informed consent is not required.

\section{References}

1. Pinto A, Eveno C, Pocard M (2017) Update on clinical trials in colorectal cancer peritoneal metastasis. Int J Hyperthermia 33(5):543-547

2. Flood M, Narasimhan V, Waters P, et al. (2020) Survival after cytoreductive surgery and hyperthermic intraperitoneal chemotherapy for colorectal peritoneal metastases: a systematic review and discussion of latest controversies. Surgeon

3. Klaver CEL, Wisselink DD, Punt CJA et al (2019) Adjuvant hyperthermic intraperitoneal chemotherapy in patients with locally advanced colon cancer (COLOPEC): a multicentre, open-label, randomised trial. Lancet GastroenterolHepatol 4(10):761-770

4. World Medical A (2013) World Medical Association Declaration of Helsinki: ethical principles for medical research involving human subjects. JAMA 310(20):2191-2194

5. Ellis L, Canchola AJ, Spiegel D, Ladabaum U, Haile R, Gomez SL (2018) Racial and ethnic disparities in cancer survival: the contribution of tumor, sociodemographic, institutional, and neighborhood characteristics. J Clin Oncol 36(1):25-33

6. Kahan BC, Rehal S, Cro S (2015) Risk of selection bias in randomised trials. Trials 16:405

Publisher's Note Springer Nature remains neutral with regard to jurisdictional claims in published maps and institutional affiliations. 